\title{
Identification of the Golden-2-like transcription factors gene family in Gossypium hirsutum
}

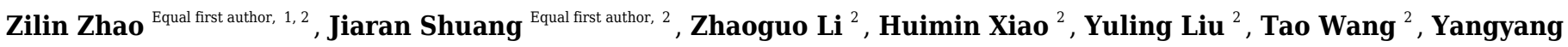 \\ Wei $^{2}$, Shoulin Hu ${ }^{1}$, Sumei Wan ${ }^{\text {Corresp., }}{ }^{\text {, Renhai Peng }}{ }^{\text {Corresp. } 1,2}$ \\ ${ }^{1}$ College of Plant Science, Tarim University, Alar, Xinjiang, China \\ 2 Anyang Institute of Technology, Anyang, Henan, China \\ Corresponding Authors: Sumei Wan, Renhai Peng \\ Email address: wansumei510@163.com, aydxprh@163.com
}

Background. Golden2-Like (GLK) transcription factors are a type of transcriptional regulator in plants. They play a pivotal role in the plant physiological activity process and abiotic stress response.

Methods. In this study, the potential function of GLK family genes in Gossypium hirsutum was studied based on genomic identification, phylogenetic analysis, chromosome mapping and cis-regulatory element prediction. Gene expression of nine key genes were analyzed by qRT-PCR experiments.

Results. Herein, we identified a total of 146 GhGLK genes in Gossypium hirsutum, which were unevenly distributed on each of the chromosomes. There were significant differences in the number and location of genes between the At sub-genome and the Dt sub-genome. According to the phylogenetic analysis, they were divided into ten subgroups, each of which had very similar number and structure of exons and introns. Some cis-regulatory elements were identified through promoter analysis, including five types of elements related to abiotic stress response, five types of elements related to phytohormone and five types of elements involved in growth and development. Based on public transcriptome data analysis, we identified nine key GhGLKs involved in salt, cold, and drought stress. The qRT-PCR results showed that these genes had different expression patterns under these stress condition, suggesting that GhGLK genes played an important role in abiotic stress response. This study laid a theoretical foundation for the screening and functional verification of genes related to stress resistance of GLK gene family in cotton. 


\section{Identification of the Golden-2-like transcription factors gene family in}

\section{Gossypium hirsutum}

3 Zilin Zhao ${ }^{1,2 \dagger}$, Jiaran Shuang ${ }^{2 \dagger}$, Zhaoguo $\mathrm{Li}^{2}$, Huimin $\mathrm{Xiao}^{2}$, Yuling $\mathrm{Liu}^{2}$, Tao Wang ${ }^{2}$, Yangyang Wei ${ }^{2}$,

4 Shoulin $\mathrm{Hu}^{1}$, Sumei Wan ${ }^{1 *}$, Renhai Peng ${ }^{1,2^{*}}$

$5 \quad{ }^{1}$ College of Plant Science, Tarim University, Alar, Xinjiang, China

$6 \quad 2$ Anyang Institute of Technology, Anyang, Henan, China

$8 \uparrow$ These authors contributed equally to this work.

9 * Co-Corresponding Author:

10 Sumei Wan

11 Alar, Xinjiang, 843300, China

12 Email address: wansumei510@163.com

13 Renhai Peng

14 Anyang, Henan, 455000, China

15 Email address: aydxprh@163.com

\section{Abstract}

Background. Golden2-Like (GLK) transcription factors are a type of transcriptional regulator in plants. They play a pivotal role in the plant physiological activity process and abiotic stress response.

Methods. In this study, the potential function of GLK family genes in Gossypium hirsutum was studied based on genomic identification, phylogenetic analysis, chromosome mapping and cisregulatory element prediction. Gene expression of nine key genes were analyzed by qRT-PCR experiments.

Results. Herein, we identified a total of 146 GhGLK genes in Gossypium hirsutum, which were unevenly distributed on each of the chromosomes. There were significant differences in the number and location of genes between the At sub-genome and the Dt sub-genome. According to the phylogenetic analysis, they were divided into ten subgroups, each of which had very similar number and structure of exons and introns. Some cis-regulatory elements were identified through promoter analysis, including five types of elements related to abiotic stress response, five types of elements related to phytohormone and five types of elements involved in growth and development. Based on public transcriptome data analysis, we identified nine key GhGLKs involved in salt, cold, and drought stress. The qRT-PCR results showed that these genes had different expression patterns under these stress condition, suggesting that $G h G L K$ genes played an important role in abiotic stress response. This study laid a theoretical foundation for the screening and functional verification of genes related to stress resistance of $G L K$ gene family in cotton.

38 Introduction 
Transcription Factors (TFs), also known as trans-acting factors, are known to activate or inhibit the transcription of downstream target genes at appropriate times. TFs affect all aspects of plant growth and development (Song et al., 2016; Ling et al., 2011). Plants are threatened by abiotic stresses during their growth, including high temperature, herbicides, heavy metals, drought, salinity, cold, pests and diseases (Nguyen et al., 2018). Crop yields are known to be seriously threatened under abiotic stress (Riechmann et al., 2000; Wray et al., 2003). Transcription factors can activate or inhibit gene transcription, affect the expression and function of their proteins, and play a significant role in plant stress response and physiological activities (Chrispeels et al., 1999; Mizoi et al., 2012).

For the first time, Golden2 (G2) has been identified that could cause maize to turn yellow (Jenkins et al., 1926). Subsequently, some studies have revealed that G2 protein was an important transcription factor in plant growth and development (Hall et al., 1998). Golden2-Like (GLK) transcription factors are members of the GARP superfamily (Riechmann et al., 2000; Xiao Y et al., 2019). Most GLK genes contain a Myb-DNA binding domain (which contains an HLH motif) and a C-terminal (GCT) box (Liu et al., 2017). In addition, some members of subgroups have conserved MYB-CC-LHEQLE domains (Qin et al., 2021).

GLK genes have been identified with different function in Physcomitrella patens, Arabidopsis thaliana, Oryza sativa, Capsicum annuum L. and Solanum lycopersicum (Hall et al., 1998; Rossini et al., 2001; Fitter et al., 2002; Waters et al., 2009; Yasumura et al., 2005). In terms of cell differentiation, functional redundancy of the GLKs is present in A. thaliana and rice (Chen et al., 2016). In corn, ZmGLK1 and ZmGLK2, a pair of homologous genes, have basically the same function and are expressed in mesophyll cells and vascular bundle sheath cells, respectively (Chang et al., 2012). These results indicate that $G L K S$ are expressed differently in different photosynthetic cells, which control the process of cell differentiation and play an important role in chloroplast development (Yasumura et al., 2005). Studies have indicated that GLK1 is mainly expressed in leaf tissue and GLK2 is mainly expressed in fruit (Chen et al., 2016). In tomato, SlGLK2 is only expressed in the fruit, and changes the content of sugar and carotenoid by regulating chloroplast development, thus affecting the fruit quality of tomato (Powell et al., 2012; Nguyen et al., 2014). In addition, some studies have found that the KNOTTED1-LIKE HOMEOBOX (KNOX) gene acts on the downstream of SlGLK2 and only affects the chloroplast development in tomato fruit but not in leaf tissue (Nadakuduti et al., 2014). All these indicate that although GLK1 and GLK2 have the same function, they have different regulatory pathways in different organs and have tissue-specific characteristics (Nguyen et al., 2014). GLKs may enrich the carbon utilization rate of plants to a certain extent and promote the growth and exploitation of plants by increasing the fixation of carbon dioxide in root (Kobayashi et al., 2014). However, the expression of $G L K s$ in leaves was significantly higher than that in roots (Fitter et al., 2002).

The $G L K s$ also play an important role in biotic stress. At present, studies on GLKs in plant disease resistance mainly focus on A. thaliana, a model system, and some studies on rice, while few studies are known on other crops (Chen et al., 2016). The overexpression of AtGLK1 enhances the resistance of $A$. thaliana infected with Fusarium graminearum, and plays a positive 
role in the tolerance to Cucumber Mosaic Virus (Savitch et al., 2007; Schreiber et al., 2011; Han et al., 2016). AtGLK1 regulates genes related to disease resistance, and its effect on different pathogens are different (Chen et al., 2016). In addition to A. thaliana, OsGLK1 in rice has also shown certain disease resistance (Nakamura et al., 2009). In addition, GLKs are also involved in hormonal response. The resistance of AtGLK1 to Hyalo-peronospora arabidopsidis Noco2 involves two signaling pathways: salicylic acid and jasmonic acid (Murmu et al., 2014). The GLK1/2-WRKY40 transcription module plays a negative regulatory role in the ABA response (Ahmad et al., 2019).

Many functions of $G L K$ genes have been studied in depth, but the functions related to abiotic stress are rarely mentioned and few studies have been reported. Research has found that some genes in the $G L K$ gene family are involved in stressresponse in maize (Liu et al., 2016). It was found that the down-regulation of the SlGLK29 gene in tomato could reduce the cold resistance (Liu, 2018). This suggests that different $G L K$ genes have distinct expression patterns under different stress condition.

Cotton is an important fiber and oil crop in the world, but its development is always threatened by abiotic stresses, including extreme temperature, drought and salt (Zhang et al., 2018; Li et al., 2021). These stresses will become more severe in the future, which will lead to lower crop yields and quality (Onaga et al., 2016). Therefore, it is necessary to identify genes related to abiotic stress resistance to assist cotton genetic improvement. So far, the identification of the $G L K$ gene family in G. hirsutum has not been reported. In our research, the GLK gene family in G. hirsutum was identified, and the subcellular localization, chromosomal distribution, gene structure and expression level of $G L K$ genes were analyzed. The results demonstrate that nine key genes responded to drought, salt and cold stress. This study provides a reference for further study of the role of GLKS in the stress response of cotton.

\section{Materials \& Methods}

\section{Identification of the GLK genes in Gossypium hirsutum.}

The genome files of G. hirsutum (TM-1 HAU_v1.1) and Theobroma cacao L. (assembly Criollo_cocoa_genome_V2) were obtained from CottonFGD (http://www.cottonfgd.org/) (Wang et al., 2019) and NCBI (https://www.ncbi.nlm.nih.gov/) ( Argout et al., 2011). The genome and protein sequences of $A$. thaliana were obtained from NCBI (https://www.ncbi.nlm.nih.gov/). The Markov Model (HMM) of PF00249.31 and PF14379.6 were downloaded from Pfam (https://pfam.xfam.org/). The TBtools v1.087 HMM Search (Chen et al., 2020) was used to identify the GLK gene family in A. thaliana, G. hirsutum and T. cacao with PF00249.31 and PF14379.6, which were most likely members of GLK gene family. Published tomato GLK protein sequences were downloaded from NCBI (https://www.ncbi.nlm.nih.gov/) (Liu, 2018). The $A$. thaliana and tomato sequences were used as query sequences to BLAST against the cotton and T. cacao genome database using TBtools (Chen et al., 2020). ProtParam tool (https://web.expasy.org/protparam/) was used to predict the physical and chemical properties of GLK proteins, including the number of amino acids, molecular weight(MW), isoelectric point(PI), and Instability index. The subcellular localization of GLKs was predicted through the 
120 WoLF PSORT (https://wolfpsort.hgc.jp/) and CELLO v.2.5 (http://cello.life.nctu.edu.tw/)

121 resources (Yu et al., 2006).

122 Phylogenetic analysis of GLK genes.

123 A phylogenetic tree was constructed the obtained GLK protein sequences of $A$. thaliana, tomato,

$124 T$. cacao and G. hirsutum. A neighbor-joining tree of GLK genes was constructed using MEGA-

$125 \mathrm{X}$ with 1000 bootstrap replications (Hall, 2013). The phylogenetic tree was drawn using

126 EvolView (He et al., 2016).

127 Analysis of the conserved motifs and gene structure.

128 The conserved sequences of GLK was identified and analyzed by the MEME website

129 (http://meme-suite.org/) (Bailey et al., 2009). The optimal width was 10 to 150, and the number

130 of motifs was 26. Everything else is set was default values. TBtools (Chen et al., 2020) was used

131 to map conserved motifs and gene structures (introns and exons), using MAST profiles from the

132 MEME website and the GFF3 profiles for each gene.

133 Chromosomal localization analysis of GLK genes.

134 Based on the genome and genome annotation files of G. hirsutum, the chromosome distribution

135 of GLK genes and their physical locations were obtained through TBtools (Chen et al., 2020).

136 Cis-regulatory elements analysis of GLK.

137 In order to analyze the promoter of $G L K$ in $G$. hirsutum and predict the function of the GLK

138 genes, $2000 \mathrm{bp}$ sequence upstream of the start codon for each gene was extracted and input into

139 the Plantcare website for analysis (http://bioinformatics.psb.ugent.be/webtools/plantcare/html/).

140 GO and KEGG enrichment analysis of GLK genes.

141 For functional enrichment analysis, GhGLKs were submitted to the omicshare tool

142 (https://www.omicshare.com/tools/) for GO and KEGG enrichment analysis.

143 Differential gene expression analysis.

144 In order to study the expression pattern of the GLK in G. hirsutum, the transcriptome sequencing 145 data of $G$. hirsutum (PRJNA490626) under cold, salt, and drought stress was downloaded from 146 NCBI (https://www.ncbi.nlm.nih.gov/). Trimmomatic (Bolger et al., 2014) was used to remove 147 the adapter and perform quality control. Reads were mapped to the genome using the hisat2 148 program (Kim et al., 2015), and then Fragments Per Kilobase of transcript per Million fragments 149 (FPKM) values of GLK genes were calculated by Cufflinks (Ghosh et al., 2016; Pollier et al., 150 2013). The expression level of $G L K$ family genes was standardized, expressed as a FPKM value, 151 and transformed in $\log _{2}$ form, and the heatmap was drawn using TBtools software (Chen et al., 152 2020).

153 Stress treatments and qRT-PCR analysis.

154 G. hirsutum acc. TM-1 planted in the experimental field of Anyang Institute of Technology was 155 selected as experimental material. The seedlings with stable growth of G. hirsutum were treated 156 with three stress: salt stress, drought stress and cold stress. The root of cotton seedlings was 157 irrigated with $250 \mathrm{mM} \mathrm{NaCl}$ solution and $18 \% \mathrm{PEG}$ solution to simulate salt stress and drought 158 stress. It was placed in a $4{ }^{\circ} \mathrm{C}$ incubator to simulate cold stress treatment. Leaves from cotton 159 seedlings with consistent growth were collected after 0 h, 1 h, 3 h, 6 h, 12 h, 24 h of above 160 stresses. All samples were immediately frozen in liquid nitrogen and stored at $-80{ }^{\circ} \mathrm{C}$ for RNA 
extraction.

Total RNA was extracted from each sample using the EASYspin Plus Plant RNA Kit (RN38, Aidlab Biotech, Beijing, China). The quality of RNA was determined by agarose gel electrophoresis and a Nanodrop2000 nucleic acid analyzer. The cDNAs were synthesized using a TranScript All-in-One First-Strand cDNA Synthesis SuperMix for qPCR (Transgen Biotech, Beijing, China). The kit used for Real-time PCR is the TransStart Top Green qPCR SuperMix kit (Transgen Biotech, Beijing, China). The instrument used is the ABI 7500 Fast Real-Time PCR system (Applied Biosystems, USA). The specific primers for these differentially expressed genes were designed using the Prime-Blast in the NCBI online database and are listed in Supplementary Table S1. Each experiment was repeated three times, and choose two groups of good data to graph. The relative gene expression levels were analyzed using the $2^{-\Delta \Delta C t}$ method (Livak \& Schmittgen, 2001).

\section{Results}

\section{Identification and analysis of basic physicochemical properties of GLK family members in} G. hirsutum.

A total of 146 GLK genes were identified from G. hirsutum, named GhGLK1-GhGLK146. The basic physical and chemical properties were also predicted and analyzed. Amino acid sequences range in length is from 140 (GhGLK27) to 826 (GhGLK139), the isoelectric point is from 5.07 ( $G h G L K 9$ ) to 9.71 (GhGLK134). The instability index refers to how stable the protein is in the test tube ( $\leq 40$, possibly stable; $>40$, possibly unstable). Prediction showed that, except for 13 genes, all other genes may be stable. According to the results of subcellular localization, all the GhGLK genes were located in the nucleus. GhGLK6, GhGLK46, GhGLK76, GhGLK128 and GhGLK139 were located in the chloroplast and nucleus. GhGLK112 and GhGLK127 were in cytoplasm and nucleus (Table S2).

\section{Phylogenetic analysis of GLK in G. hirsutum.}

Using tomato, A. thaliana and T. cacao GLK protein sequences as reference, a rootless phylogenetic tree of GLK protein in G. hirsutum was constructed (Fig. 1). As the results shown, $146 G L K$ genes of $G$. hirsutum were divided in ten subfamilies. At the same time, the number of GLKs in G. hirsutum was much higher than that in A. thaliana, T. cacao and tomato.

\section{Gene structure and protein conserved motif analysis of GLK in G. hirsutum.}

The motif of each protein, namely the conserved element, was analyzed by MEME. A total of 26 possible motifs were identified. Motif 1 was included in all GhGLKs , and it was conservative for GhGLKs. These motifs differ between subfamilies, but were conserved within each subfamily. For example, motifs 17 and 18 were found only in subfamily D and E, respectively. Some subfamilies had very conservative motifs. For example, all members of the $\mathrm{C}$ subfamily had motif 5, 3, 8, 2, 1, 14, 10. Predictions about exons and introns of the $G L K$ genes were shown in the Fig. 2. The number of exons was at least one and at most eleven. Except for GhGLK87, the other members of the D subfamily had one exon, and all members of the C subfamily had 11 exons. Most of the other members had between 4 and 7 introns. The number of exons and introns varied among different subfamilies, but in the same subfamily, the exon-intron structure of most 
201

202

203

204

205

206

207

208

209

210

211

212

213

214

215

216

217

218

219

220

221

222

223

224

225

226

227

228

229

230

231

232

233

234

235

236

237

238

239

240

241

members showed great similarity.

Chromosomal localization analysis of GLK family genes in G. hirsutum.

Based on a physical map of the GLK family members of G. hirsutum, 144 of 146 GLK genes were located on 26 chromosomes (Fig. 3). The other two genes were located on the scaffold. Among the chromosome-located genes, seventeen genes were located on chromosome 5. There were sixteen genes located on chromosomes 8 and 12, only six genes were located on chromosome 2 (Fig. 4). There were significant differences in At sub-genome and Dt sub-genome about the number and location of genes. For example, there were two genes on chromosome A02 and four genes on chromosome D02. This may be due to the fact that G. hirsutum is a tetraploid cotton species, which is a hybrid between an A-genome-like Gossypium herbaceum and a Dgenome-like Gossypium raimondii.

\section{Analysis of promoters of GLK genes.}

The Plantcare was used to analyze the sequence of $2000 \mathrm{bp}$ upstream of the promoter region of GhGLK genes, and the found cis-regulatory elements were shown in Fig. 5. We found several cis-regulatory elements in stress response, which were associated with anaerobic induction, defense and stress response, drought, cold, and wound, respectively. Meanwhile, we also found a number of hormone-responsive cis-regulatory elements, which were associated with abscisic acid (ABA), auxin (IAA), gibberellin (GA), salicylic acid (SA), and methyl jasmonate (MeJA). Among all cis-regulatory elements, the number of elements related to light response was the largest and the distribution was the widest. In addition, we had identified cis-regulatory elements involved in cell cycle regulation, circadian rhythm, down regulation of photosin expression, and regulation of flavonoid biosynthesis genes. In summary, different types and quantities of cisregulatory elements were distributed in different GhGLK promoters of cotton. According to the results, it was speculated that under environmental stress, the cis-regulatory elements leaded to the expression of $G h G L K$ genes, thereby enhancing the resistance to environmental stress.

\section{GO and KEGG enrichment analysis of GLK in G. hirsutum.}

In order to further understand the function of $G h G L K s$, we carried out functional enrichment annotation of gene ontology (GO) and kyoto encyclopedia of genes and genomes (KEGG). The results improved our accurate understanding of gene function, including many significantly enriched terms (Fig. 6, Table S2). The GO enrichment analysis of GhGLKs was divided into 3 categories, including biological processes, molecular functions and cellular components, of which the biological process had the largest number of $13 \mathrm{GO}$ entries, followed by molecular functions and cellular components. The biological process was mainly concentrated in four subclasses: cellular process, metabolic process, regulation of biological process and biological regulation. The molecular function mainly included binding, nucleic acid binding transcription factor activity and catalytic activity. The cellular components were mainly organelle subclasses. KEGG pathway enrichment analysis of GLK family genes revealed two pathways, RNA transport and plant hormone signal transduction respectively. To sum up, the functional enrichment analysis results confirmed the functions of GhGLKs in many biological processes, which were related to plant growth and development, plant hormone signal transduction and so on. 
242 Expression analysis of GLK gene in G. hirsutum under different abiotic stresses.

243 To investigate the response of $G h G L K$ to adversity stress, we analyzed the expression levels of 244146 GhGLKs under different adversity conditions. The results showed that the expression of $245 G h G L K$ genes altered under salt, drought and cold stress, which revealed that $G h G L K s$ were 246 involved in the regulation of adversity stress (Fig. 7). According to the above results, we selected 247 nine key genes with differentially expressed GhGLKs and analyzed the expression pattern by 248 qRT-PCR (Fig. 8). 
282

283

284

285

286

287

288

289

290

291

292

293

294

295

296

297

298

299

300

301

302

303

304

305

306

307

308

309

310

311

312

313

314

315

316

317

318

319

320

321

322

and isoelectric point distribution range were very large. This might be due to the large-scale replication of the upland cotton genome and the large number of upland cotton GhGLK genes. This study predicted the location of G. hirsutum GLKs in the cell, and found that some genes were located in the chloroplast. Previous studies had shown that the GLK gene is related to the growth and development of the chloroplast. This result was also consistent with the results of previous studies.

We analyzed the phylogenetic relationship of G. hirsutum GLKs, and constructed a phylogenetic tree of tomato, A. thaliana and G. hirsutum. The results showed that the GLK gene of $G$. hirsutum can be divided into 11 subfamilies, while tomato and corn were divided into 6 subfamilies (Liu, 2018). This might be due to the fact that the number of GLK genes in cotton was larger than that of $G L K$ in tomato, resulting in a more precise and accurate sub-family classification. Some $G h G L K$ and tomato $G L K$ were homologous, and the results of this study indicated that there was a close evolutionary relationship between $G L K$ genes. At the same time, we found that $G h G L K$ gathered in the same subfamily had similar motif distribution patterns, motif positions and lengths.

The gene structure analysis also showed that the $G L K$ gene had strong evolutionary conservation. $G L K$ genes in the same subgroup had similar number of exons/introns and length of exons. The results of previous studies showed that the structure of the exons and introns of each subfamily of $G L K$ genes in tomato showed a large similarity, and the number of introns of most members was between 4 and 7. The results were the same in G. hirsutum, except that there are up to 11 exons in upland cotton and up to 7 in tomato(Liu, 2018).

In the G. hirsutum GLK gene family, 144 genes were located on 26 chromosomes of group $\mathrm{A}$ and $\mathrm{D}$, and the other 2 genes were located on scaffolds of unknown chromosomes. Among them, there were $70 G L K$ genes on chromosomes of group A and chromosomes of group D. There were $74 G L K$ genes on it. The distribution of the $G L K$ gene in $G$. hirsutum was uneven on the chromosomes, but the distribution of the $G L K$ gene on the two homologous chromosomes was indeed the same.

Many cis-elements in $G h G L K$ promoter were related to biotic and abiotic stress. In order to further understand the function of $G h G L K$ under different environmental stresses, we analyzed the expression patterns of $9 G h G L K$ under different environmental stresses by qRT-PCR. This showed that most selected GhGLKs respond to abiotic stresses, including drought, cold, and salt. In previous studies, it was found that the expression pattern of $Z m G L K 3$ gene in maize was upregulated under drought and salt stress (Liu et al., 2016); the expression of $S l G L K 7$ in tomato was up-regulated under three stresses (Liu, 2018). The expression of SlGLK15 and SlGLK37 genes in tomato decreased first and then increased during cold stress and salt stress, and these two genes belonged to the same subfamily(Liu, 2018). In our results, we found that the two genes GhGLK55 and GhGLK120 were located in the same subgroup, and they were in the same subgroup as SlGLK15 and SlGLK37 in tomato. These two genes in G. hirsutum also decreased firstly and then increased under cold and salt stress, indicating that the orthologous genes they should have similar expression patterns in the face of adversity and coercion. We also found that the differentially expressed genes all have anaerobic-induced and light-responsive cis-acting 
323

324

325

326

327

328

329

330

331

332

333

334

335

336

337

338

339

340

341

342

343

344

345

346

347

348

349

350

351

352

353

354

355

356

357

358

359

360

elements, and they all contained at least one abiotic stress-responsive element. GhGLK120 had the most hormone-responsive elements.

\section{Conclusions}

In this study, 146 GhGLK genes were identified in G. hirsutum. Based on the analysis of their physical and chemical properties, subcellular localization, phylogenetic relationship, distribution of cis-regulatory elements, we identified nine key GhGLKs involved in salt, cold, and drought stress. The qRT-PCR results of these genes in three stress responses showed that GhGLKs played an important role in abiotic stress responses in cotton. It was of great significance to make full use of its resistance germplasm resources and provided a theoretical basis for further mining of cotton resistance genes.

\section{References}

Ahmad R, Liu Y, Wang TJ, Meng Q, Yin H, Wang X, Wu Y, Nan N, Liu B, Xu ZY. 2019. GOLDEN2-LIKE Transcription Factors Regulate WRKY40 Expression in Response to Abscisic Acid. Plant Physiology 179(4):1844-1860. DOI 10.1104/pp.18.01466.

Argout X, Salse J, Aury JM, Guiltinan MJ, Droc G, Gouzy J, Allegre M, Chaparro C, Legavre T, Maximova SN, Abrouk M, Murat F, Fouet O, Poulain J, Ruiz M, Roguet Y, Rodier-Goud M, Barbosa-Neto JF, Sabot F, Kudrna D, Ammiraju JS, Schuster SC, Carlson JE, Sallet E, Schiex T, Dievart A, Kramer M, Gelley L, Shi Z, Bérard A, Viot C, Boccara M, Risterucci AM, Guignon V, Sabau X, Axtell MJ, Ma Z, Zhang Y, Brown S, Bourge M, Golser W, Song X, Clement D, Rivallan R, Tahi M, Akaza JM, Pitollat B, Gramacho K, D'Hont A, Brunel D, Infante D, Kebe I, Costet P, Wing R, McCombie WR, Guiderdoni E, Quetier F, Panaud O, Wincker P, Bocs S, Lanaud C. 2011. The genome of Theobroma cacao. Nature Genetics 43(2):101-8. DOI 10.1038/ng.736.

Bailey TL, Boden M, Buske FA, Frith M, Grant CE, Clementi L, Ren J, Li WW, Noble WS. 2009. MEME SUITE: tools for motif discovery and searching. Nucleic Acids Research 37(Web Server issue):W202-8. DOI 10.1093/nar/gkp335.

Bolger AM, Lohse M, Usadel B. 2014. Trimmomatic: a flexible trimmer for Illumina sequence data. Bioinformatics 30(15):2114-20. DOI 10.1093/bioinformatics/btu170.

Chang YM, Liu WY, Shih AC, Shen MN, Lu CH, Lu MY, Yang HW, Wang TY, Chen SC, Chen SM, Li WH, Ku MS. 2012. Characterizing regulatory and functional differentiation between maize mesophyll and bundle sheath cells by transcriptomic analysis. Plant Physiology 160(1):165-77. DOI 10.1104/pp.112.203810.

Chen C, Chen H, Zhang Y, Thomas HR, Frank MH, He Y, Xia R. 2020. TBtools: An Integrative Toolkit Developed for Interactive Analyses of Big Biological Data. Molecular Plant 13(8):1194-1202. DOI 10.1016/j.molp.2020.06.009.

Chen M, Ji M, Wen B, Liu L, Li S, Chen X, Gao D, Li L. 2016. GOLDEN 2-LIKE Transcription Factors of Plants. Frontiers in Plant Science 7:1509. DOI 10.3389/fpls.2016.01509. 
361

362

Chrispeels HE, Oettinger H, Janvier N, Tague BW. 2000. AtZFP1, encoding Arabidopsis thaliana $\mathrm{C} 2 \mathrm{H} 2$ zinc-finger protein 1, is expressed downstream of photomorphogenic activation. Plant Molecular Biology 42(2):279-90. DOI 10.1023/a:1006352809700.

Fitter DW, Martin DJ, Copley MJ, Scotland RW, Langdale JA. 2002. GLK gene pairs regulate chloroplast development in diverse plant species. The Plant Journal 31(6):713-27. DOI 10.1046/j.1365-313x.2002.01390.x.

Ghosh S, Chan CK. 2016. Analysis of RNA-Seq Data Using TopHat and Cufflinks. Methods in molecular biology 1374:339-61. DOI 10.1007/978-1-4939-3167-5_18.

Hall LN, Rossini L, Cribb L, Langdale JA. 1998. GOLDEN 2: a novel transcriptional regulator of cellular differentiation in the maize leaf. Plant Cell 10(6):925-36. DOI 10.1105/tpc.10.6.925.

Hall BG. 2013. Building phylogenetic trees from molecular data with MEGA. Molecular Biology Evolution 30(5):1229-35. DOI 10.1093/molbev/mst012.

Han XY, Li PX, Zou LJ, Tan WR, Zheng T, Zhang DW, Lin HH. 2016. GOLDEN2-LIKE transcription factors coordinate the tolerance to Cucumber mosaic virus in Arabidopsis. Biochemical and Biophysical Research Communications 477(4):626-632. DOI 10.1016/j.bbrc.2016.06.110.

He Z, Zhang H, Gao S, Lercher MJ, Chen WH, Hu S. 2016. Evolview v2: an online visualization and management tool for customized and annotated phylogenetic trees. Nucleic Acids Research 44(W1):W236-41. DOI 10.1093/nar/gkw370.

Jenkins M.T. 1926. A second gene producing golden plant color in maize. The American Naturalist 60(670):484-488.

Kim D, Langmead B, Salzberg SL. 2015. HISAT: a fast spliced aligner with low memory requirements. Nature Methods 12(4):357-60. DOI 10.1038/nmeth.3317.

Kobayashi K, Baba S, Obayashi T, Sato M, Toyooka K, Keränen M, Aro EM, Fukaki H, Ohta H, Sugimoto K, Masuda T. 2012. Regulation of root greening by light and auxin/cytokinin signaling in Arabidopsis. Plant Cell 24(3):1081-95. DOI 10.1105/tpc.111.092254.

Li Z, Liu Z, Wei Y, Liu Y, Xing L, Liu M, Li P, Lu Q, Peng R. 2021. Genome-wide identification of the MIOX gene family and their expression profile in cotton development and response to abiotic stress. PLoS One 16(7):e0254111. DOI 10.1371/journal.pone.0254111.

Ling J, Jiang W, Zhang Y, Yu H, Mao Z, Gu X, Huang S, Xie B. 2011. Genome-wide analysis of $W R K Y$ gene family in Cucumis sativus. BMC Genomics 12:471. DOI 10.1186/1471-2164-12-471.

Liu F, Xu Y, Han G, Zhou L, Ali A, Zhu S, Li X. 2016. Molecular Evolution and Genetic Variation of G2-Like Transcription Factor Genes in Maize. PLoS One 11(8):e0161763. DOI 10.1371/journal.pone.0161763.

Liu G, Zhao TT, Yang HH, Zhang DY, Jiang JB, Li JF and Xu XY. 2016. Research progress on transcriptomics in tomato. Jiyinzuxue Yu Yingyong Shengwuxue (Genomics and Applied Biology) 35(10):2802-2807. 
401

402

403

404

405

406

407

408

409

410

411

412

413

414

415

416

417

418

419

420

421

422

423

424

425

426

427

428

429

430

431

432

433

434

435

436

437

438

439

440

Liu JF. 2018. Bioinformatics analysis of tomato G2-like transcription factor family and identification of resistance-related genes. D. Agriculture. Thesis. College of Horticulture \& Landscape Architecture.

Liu JF, Zhang J, Li H, Zhao TT, and Li JF, 2017, Research progress of plant GOLDEN2Like transcription factor, Fenzi Zhiwu Yuzhong (Molecular Plant Breeding) 15(10): 39493956.

Livak KJ, Schmittgen TD. 2001. Analysis of relative gene expression data using real-time quantitative PCR and the 2 (-Delta Delta C(T)) Method. Methods 25(4):402-8. DOI 10.1006/meth.2001.1262.

Mizoi J, Shinozaki K, Yamaguchi-Shinozaki K. 2012. AP2/ERF family transcription factors in plant abiotic stress responses. Biochim Biophys Acta 1819(2):86-96. DOI 10.1016/j.bbagrm.2011.08.004.

Murmu J, Wilton M, Allard G, Pandeya R, Desveaux D, Singh J, Subramaniam R. 2014. Arabidopsis GOLDEN2-LIKE (GLK) transcription factors activate jasmonic acid (JA)dependent disease susceptibility to the biotrophic pathogen Hyaloperonospora arabidopsidis, as well as JA-independent plant immunity against the necrotrophic pathogen Botrytis cinerea. Molecular Plant Pathology 15(2):174-84. DOI 10.1111/mpp.12077.

Nadakuduti SS, Holdsworth WL, Klein CL, Barry CS. 2014. KNOX genes influence a gradient of fruit chloroplast development through regulation of GOLDEN2-LIKE expression in tomato. The Plant Journal 78(6):1022-33. DOI 10.1111/tpj.12529.

Nakamura H, Muramatsu M, Hakata M, Ueno O, Nagamura Y, Hirochika H, Takano M, Ichikawa H. 2009. Ectopic overexpression of the transcription factor $O s G L K 1$ induces chloroplast development in non-green rice cells. Plant Cell Physiology 50(11):1933-49. DOI 10.1093/pcp/pcp138.

Nguyen CV, Vrebalov JT, Gapper NE, Zheng Y, Zhong S, Fei Z, Giovannoni JJ. 2014. Tomato GOLDEN2-LIKE transcription factors reveal molecular gradients that function during fruit development and ripening. Plant Cell 26(2):585-601. DOI 10.1105/tpc.113.118794.

Nguyen HC, Lin KH, Ho SL, Chiang CM, Yang CM. 2018. Enhancing the abiotic stress tolerance of plants: from chemical treatment to biotechnological approaches. Plant Physiology 164(4):452-466. DOI 10.1111/ppl.12812.

Onaga G, Wydra K. 2016. Advances in Plant Tolerance to Abiotic Stresses. Plant Genomics http://dx.doi.org/10.5772/64350.

Pollier J, Rombauts S, Goossens A. 2013. Analysis of RNA-Seq data with TopHat and Cufflinks for genome-wide expression analysis of jasmonate-treated plants and plant cultures. Methods in molecular biology 1011:305-15. DOI 10.1007/978-1-62703-414-2_24.

Powell AL, Nguyen CV, Hill T, Cheng KL, Figueroa-Balderas R, Aktas H, Ashrafi H, Pons C, Fernández-Muñoz R, Vicente A, Lopez-Baltazar J, Barry CS, Liu Y, Chetelat R, Granell A, Van Deynze A, Giovannoni JJ, Bennett AB. 2012. Uniform ripening encodes a Golden 2-like transcription factor regulating tomato fruit chloroplast development. Science 336(6089):1711-5. DOI 10.1126/science.1222218. 
Qin M, Zhang B, Gu G, Yuan J, Yang X, Yang J, Xie X. 2021. Genome-Wide Analysis of the G2-Like Transcription Factor Genes and Their Expression in Different Senescence Stages of Tobacco (Nicotiana tabacum L.). Frontiers in Genetics 31(12):626352. DOI 10.3389/fgene.2021.626352.

Rossini L, Cribb L, Martin DJ, Langdale JA. 2001. The maize golden2 gene defines a novel class of transcriptional regulators in plants. Plant Cell 13(5):1231-44. DOI 10.1105/tpc.13.5.1231.

Riechmann JL, Heard J, Martin G, Reuber L, Jiang C, Keddie J, Adam L, Pineda O, Ratcliffe OJ, Samaha RR, Creelman R, Pilgrim M, Broun P, Zhang JZ, Ghandehari D, Sherman BK, Yu G. 2000. Arabidopsis transcription factors: genome-wide comparative analysis among eukaryotes. Science 290(5499):2105-10. DOI 10.1126/science.290.5499.2105.

Savitch LV, Subramaniam R, Allard GC, Singh J. 2007. The GLK1 'regulon' encodes disease defense related proteins and confers resistance to Fusarium graminearum in Arabidopsis. Biochemical and Biophysical Research Communications 359(2):234-8. DOI 10.1016/j.bbrc.2007.05.084.

Schreiber KJ, Nasmith CG, Allard G, Singh J, Subramaniam R, Desveaux D. 2011. Found in translation: high-throughput chemical screening in Arabidopsis thaliana identifies small molecules that reduce Fusarium head blight disease in wheat. Mol Plant Microbe Interact 24(6):640-8. DOI 10.1094/MPMI-09-10-0210.

Song A, Wu D, Fan Q, Tian C, Chen S, Guan Z, Xin J, Zhao K, Chen F. 2016.

Transcriptome-wide identification and expression profiling analysis of chrysanthemum trihelix transcription factors. International Journal of Molecular Sciences 17(2):198. DOI 10.3390/ijms17020198.

Wang M, Tu L, Yuan D, Zhu D, Shen C, Li J, Liu F, Pei L, Wang P, Zhao G, Ye Z, Huang H, Yan F, Ma Y, Zhang L, Liu M, You J, Yang Y, Liu Z, Huang F, Li B, Qiu P, Zhang Q, Zhu L, Jin S, Yang X, Min L, Li G, Chen LL, Zheng H, Lindsey K, Lin Z, Udall JA, Zhang X. 2019. Reference genome sequences of two cultivated allotetraploid cottons, Gossypium hirsutum and Gossypium barbadense. Nature Genetics 51(2): 224-229. DOI 10.1038/s41588-018-0282-x.

Waters MT, Wang P, Korkaric M, Capper RG, Saunders NJ, Langdale JA. 2009. GLK transcription factors coordinate expression of the photosynthetic apparatus in Arabidopsis. Plant Cell 21(4):1109-28. DOI 10.1105/tpc.108.065250.

Wray GA, Hahn MW, Abouheif E, Balhoff JP, Pizer M, Rockman MV, Romano LA. 2003. The evolution of transcriptional regulation in eukaryotes. Molecular Biology and Evolution 20(9):1377-419. DOI 10.1093/molbev/msg140.

Xiao Y, You S, Kong W, Tang Q, Bai W, Cai Y, Zheng H, Wang C, Jiang L, Wang C, Zhao Z, Wan J. 2019. A GARP transcription factor anther dehiscence defected 1 (OsADD1) regulates rice anther dehiscence. Plant Molecular Biology 101(4-5): 403-414. DOI 10.1007/s11103-019-00911-0.

Yu CS, Chen YC, Lu CH, Hwang JK. 2006. Prediction of protein subcellular localization. Proteins 64(3):643-51. DOI 10.1002/prot.21018. 
482 Zhang B, Liu J, Yang ZE, Chen EY, Zhang CJ, Zhang XY, Li FG. 2018. Genome-wide 483 analysis of GRAS transcription factor gene family in Gossypium hirsutum L. BMC Genomics 484 19(1):348. DOI 10.1186/s12864-018-4722-x. 
Figure 1

Phylogenetic analysis of GLK proteins from Gossypium hirsutum, Theobroma cacao L., tomato and Arabidopsis thaliana.

The blue marks are members of the tomato GLK family; The green circles are members of the A. thaliana GLK family; The purple squares are members of the $T$. cacao GLK family; The yellow star is the GLK member of cotton subgroup A, and the red triangle is the D subgroup. The blue squares are GLK genes located on the scaffolds. 


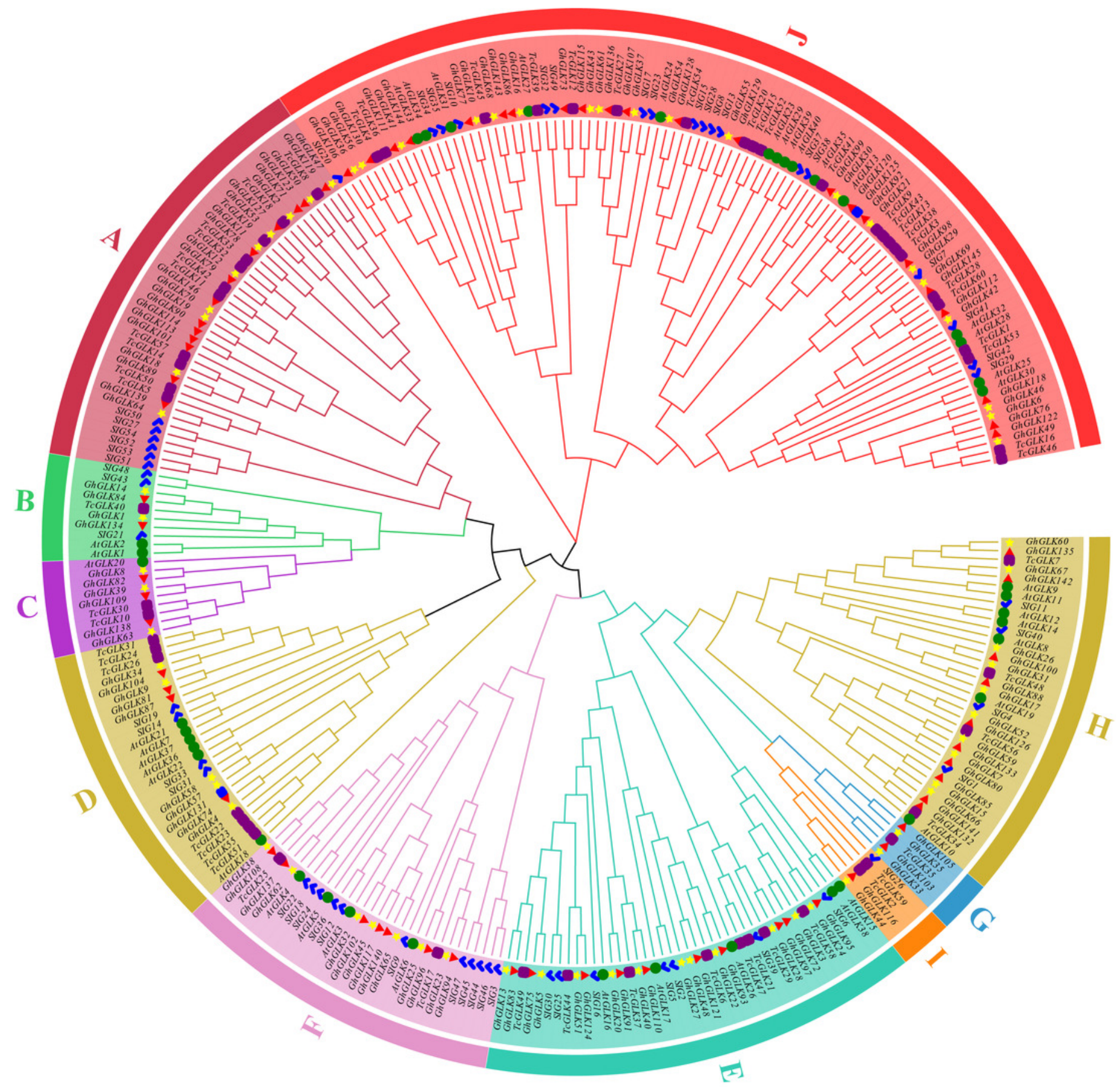


Figure 2

The conserved motif and exon-intron structure of GLK genes in Gossypium hirsutum.

(a)Phylogenetic analysis of GhGLK genes. (b)Analysis of conserved motif of GhGLK protein sequences. Different motifs are shown in a specific color. (c) Intron and exon analysis of GhGLK genes. Exons and introns are represented by yellow boxes and thin lines, respectively. The UTR is shown in a green box. 


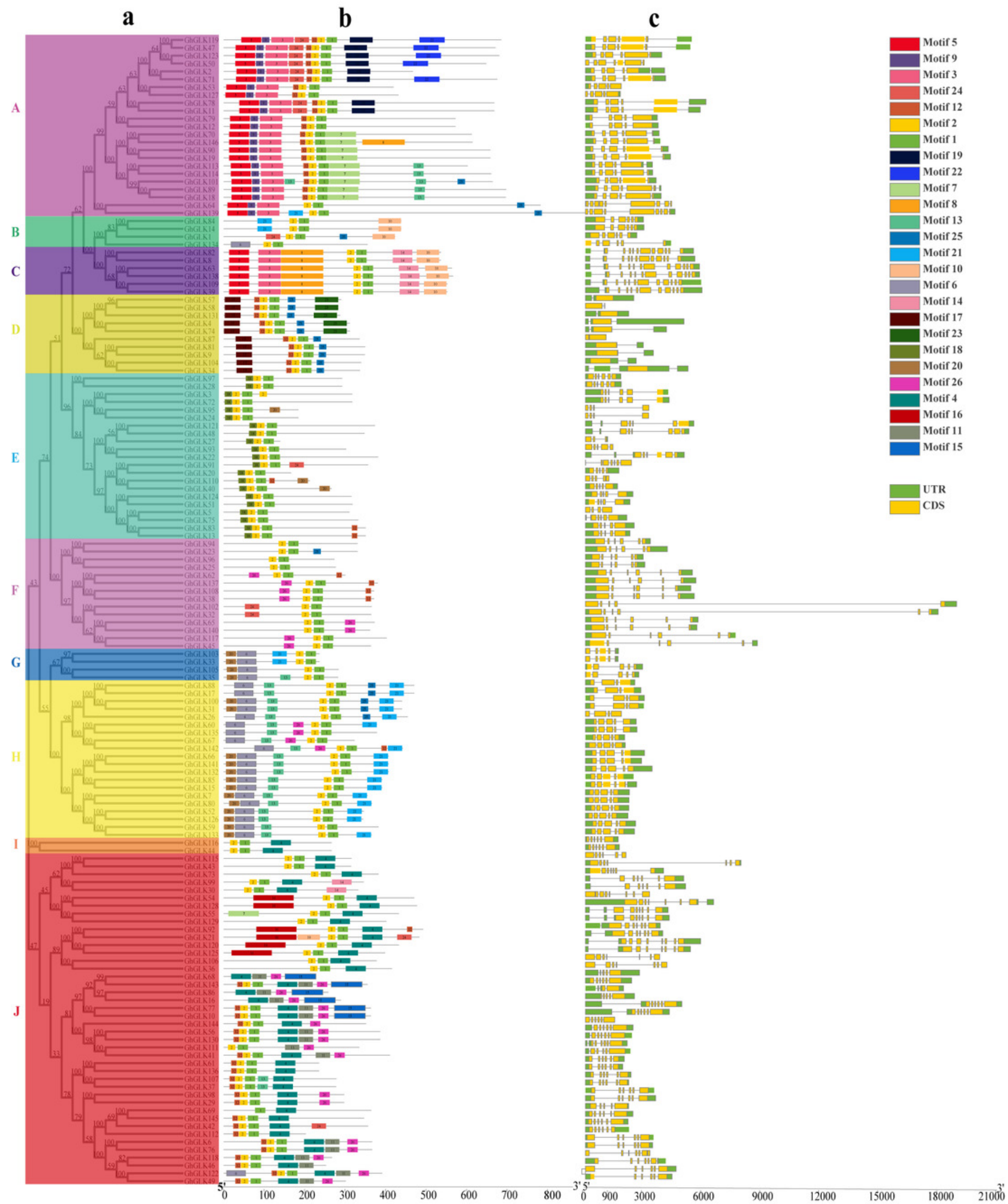


Figure 3

Chromosome distribution statistics of GhGLK genes.

Blue represents the number of genes locate on each chromosome of subgroup $A$, while red represents the number of genes locate on each chromosome of subgroup $D$.

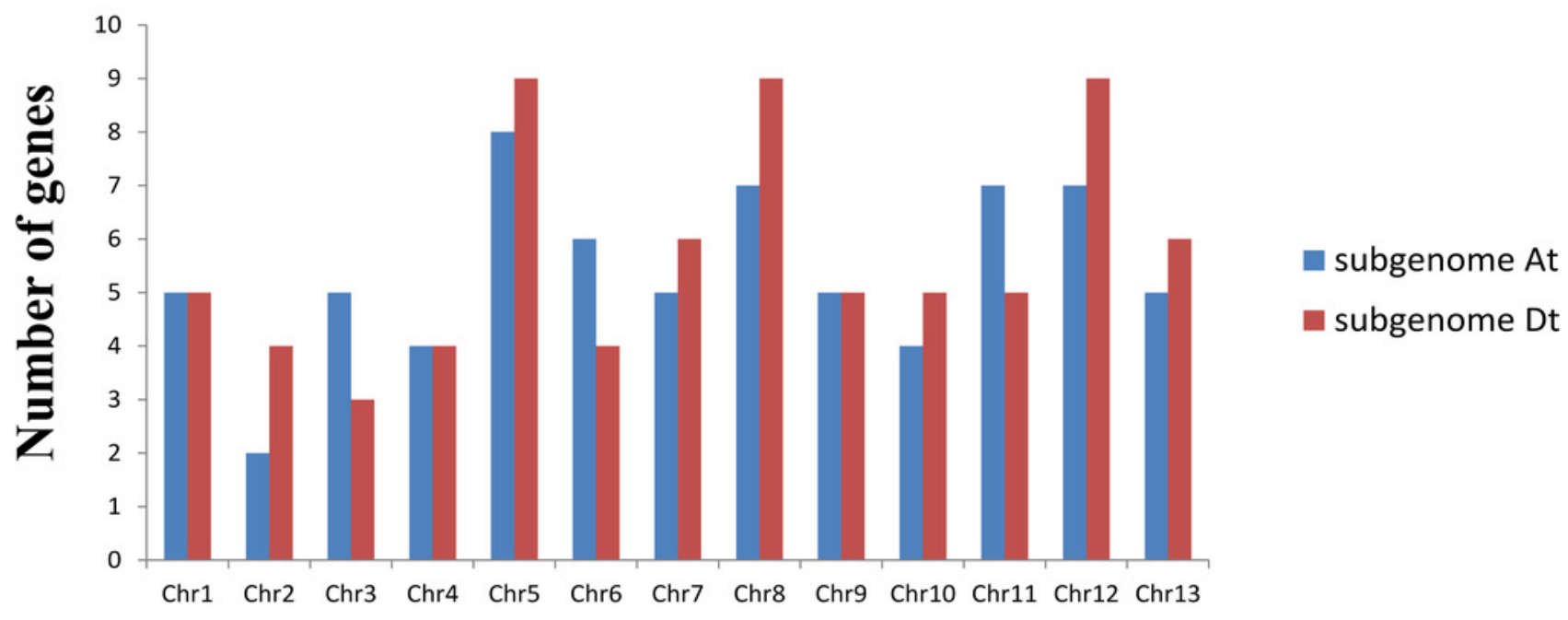

Chromosome 
Figure 4

Location of GhGLK genes on chromosome.

GhGLKs are located on 26 chromosomes of Gossypium hirsutum, and two genes are located on scaffold. Chromosome names are shown on the left and gene names are shown on the right.

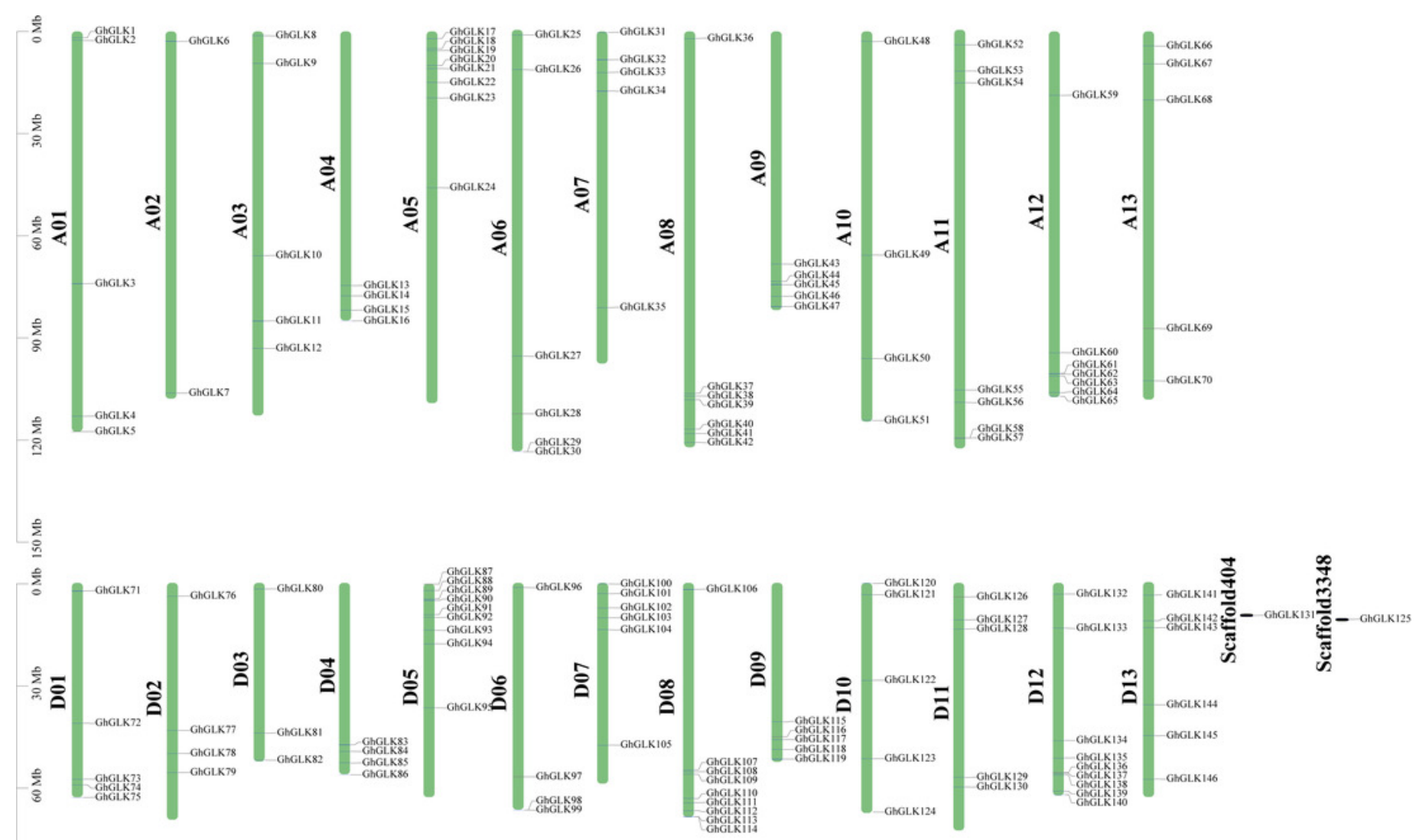


Figure 5

Analysis of cis-regulatory elements in promoters of GhGLK genes.

Cis-regulatory elements with the same function are shown in the same color. 


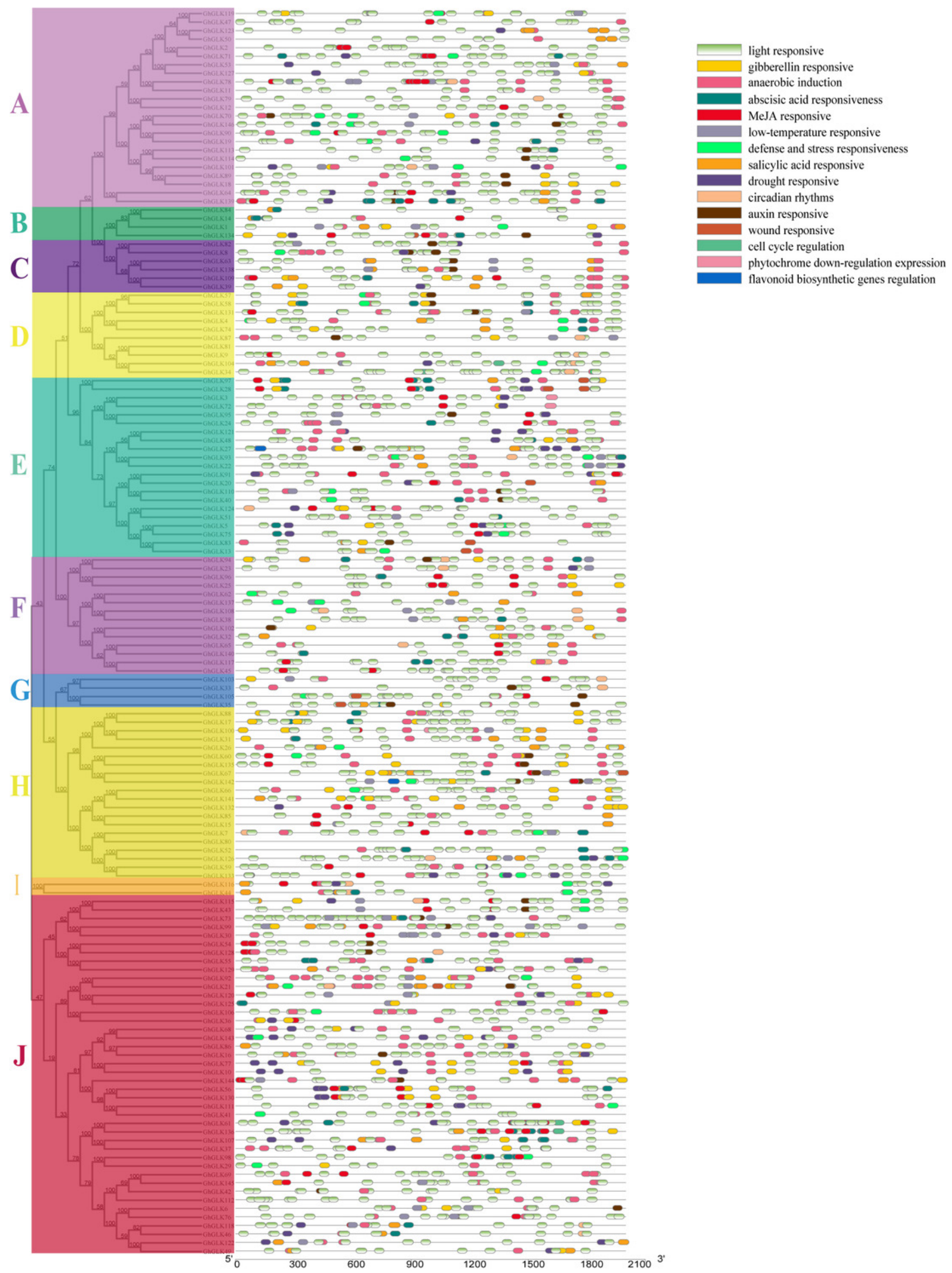


Figure 6

GO and KEGG enrichment analysis of GhGLKs.

(A)The numbers of level2 GO terms. (B)KEGG pathway enrichment analysis of GhGLKs. 
A

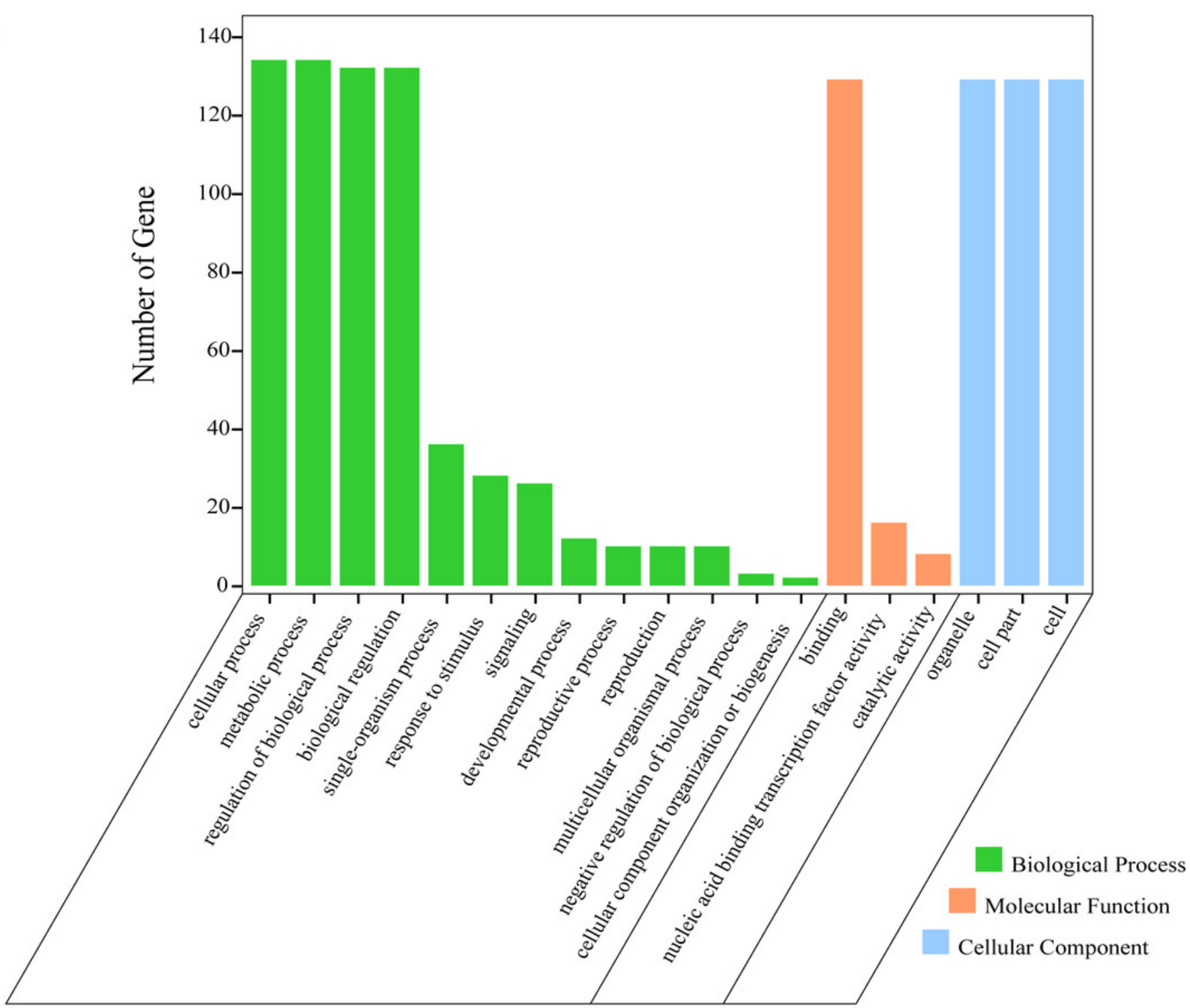

GeneNumber

B

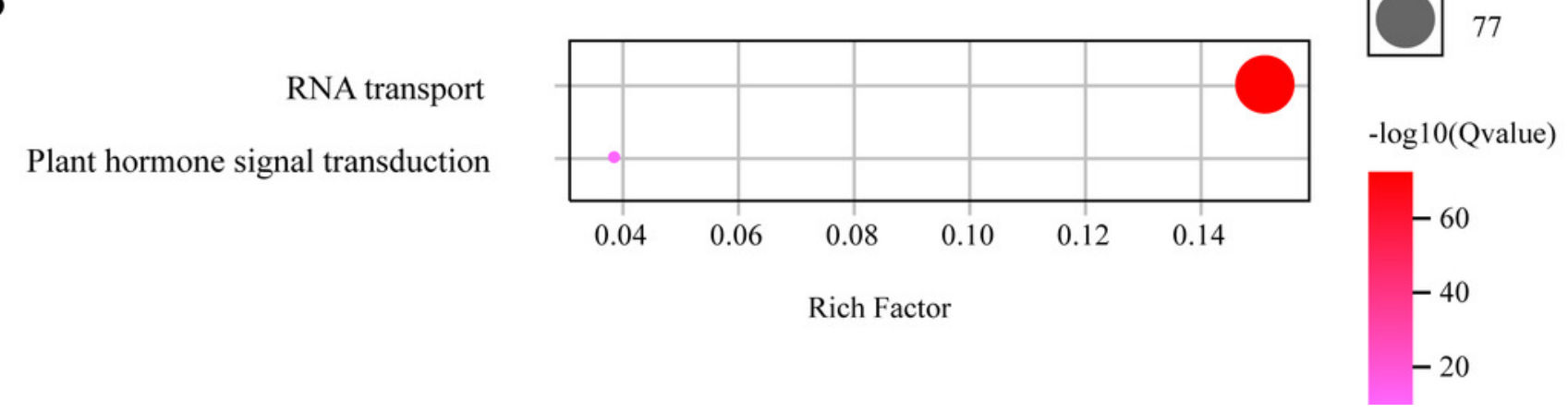




\section{Figure 7}

\section{Differentially expressed genes of GhGLKs under cold, $\mathrm{NaCl}$ and PEG stress.}

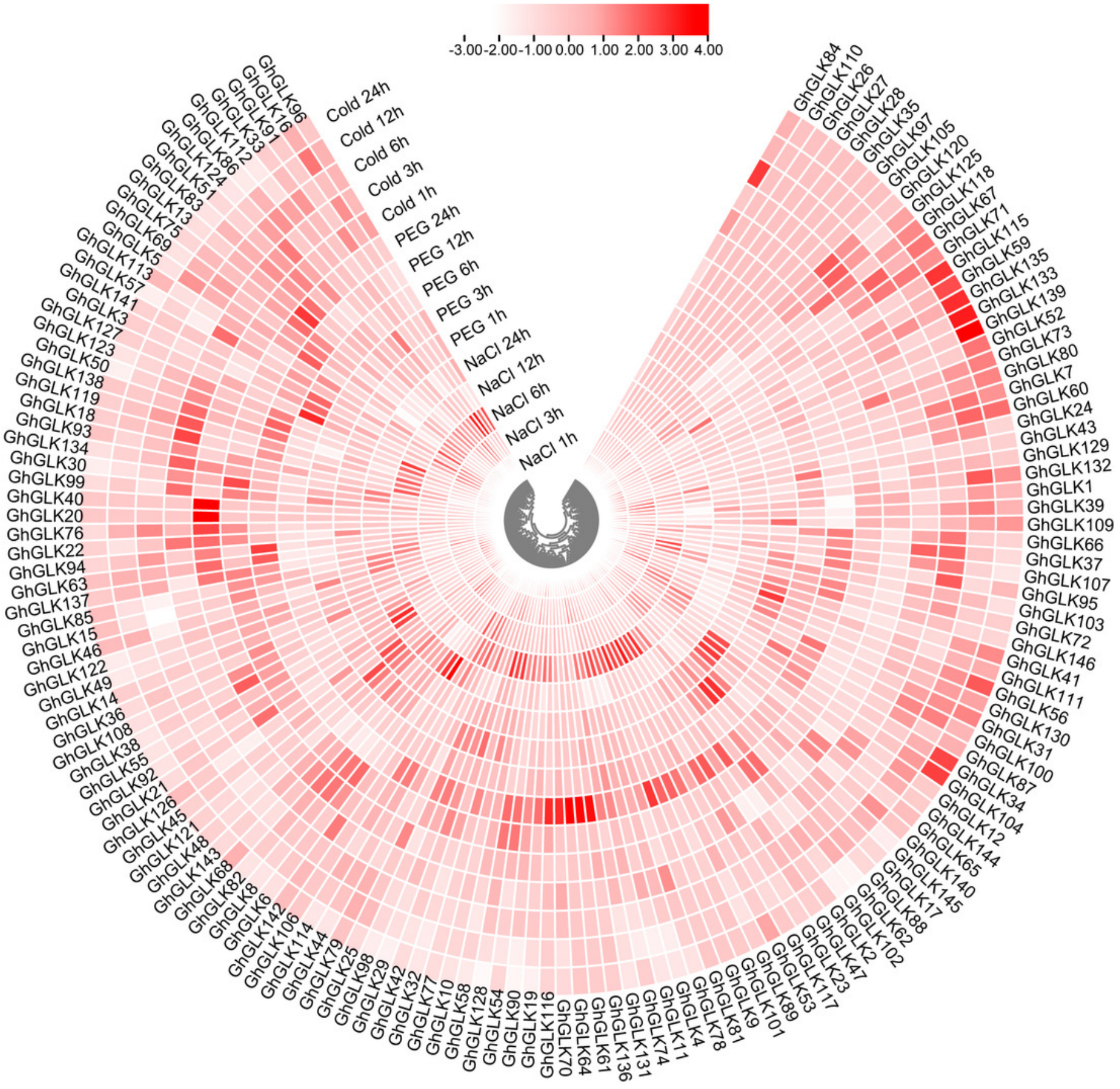


Figure 8

Expression patterns of GhGLK gene family members under cold, drought, and salt stress.

(A)Expression patterns analysis of GhGLKs under salt stress. (B)Expression patterns analysis of GhGLKs under drought stress. (C)Expression patterns analysis of GhGLKs under cold stress.

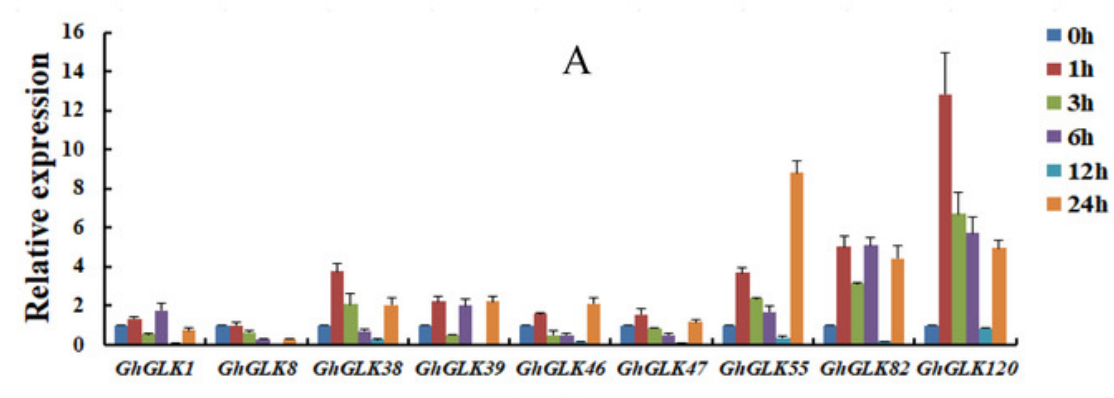

Salt Stress
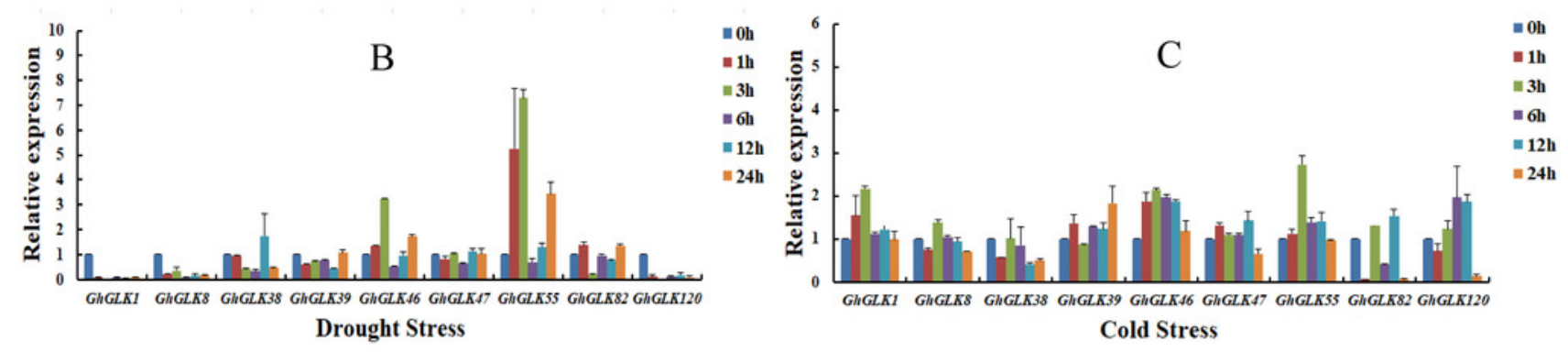